\title{
An on-line glossary as a way to foster the construction of a common culture among urban experts, stakeholders and decision- makers
}

\author{
Jacques Teller LEMA, University of Liège, Belgium
}

\section{Accepted 4 December 2001}

\begin{abstract}
Negotiation and public participation are a key aspect of present urban development projects. Argumentation support tools, based on the Toulmin logic, have often been presented as a way to increase the accountability of the debates between the different urban actors. It should still be assumed that any collaborative decision making requires some common culture to make the dialogue possible and constructive. Such a common understanding of basic terms, references and methods is often missing in present-day urban disputes, especially when complex issues such as cultural heritage are at stake. The present paper discusses the relevancy of web glossaries as a way to build up such a common culture among urban experts, stakeholders and decision makers.
\end{abstract}

Key words: collaborative decision making, cultural heritage, urban development

\section{Introduction}

The networks of buildings, monuments, streets, squares and parks uniquely define European towns and cities. These are the palpable, even if unconscious, cultural horizon for those who live and work in them. Built heritage, as well as satisfying the mundane requirements of shelter and comfort, brings essential stability and richness to our lives and provides the singular, evolving expression of the achievements, values and identity of specific communities. Quite typically municipalities and town councils are the public bodies politically responsible for the conservation of this important heritage. Nevertheless they cannot claim to control nor decide on all the transformations that contribute to its development. They are caught between legal responsibilities enshrined in European and national legislation and responsibilities towards their constituents. Furthermore, given their acknowledged lack of financial means and political leadership, they are required to embrace an increasing diversity of participants - heritage protection bodies, private developers, global corporations, special interest groups and citizens - in the decision-making processes that mould the future urban landscapes.

Given this moving context, the public authorities' role has dramatically changed in its scope. It is no longer restricted to the production of rules nor limited to direct financial support as it was in the past. These two aspects obviously remain present, especially when dealing with major and trusted heritage, but the regulating role of public authorities has been extended to more dynamic aspects. The public authorities have often to act as a facilitator,

Address for correspondence: Jacques Teller, LEMA, University of Liège, 1 Chemin de Chevreuils, B 52, 4000 Liège, Belgium. E-mail: jacques.teller@ulg.ac.be 
between convergent yet fragmented interests, as a mediator, between private actors and higher political levels, or even as an effector, between general rules and their local transposition (Teller, 2001). These new roles are best held by municipalities that have a keen knowledge of the local situation and close relations with the stakeholders, but they obviously require a strong institutional and methodological framework to assure the best communication between the involved people. Especially in those small and medium size cities where skills and means are often scarce despite a sometimes important cultural heritage to keep alive.

The management of this emerging political situation requires the availability of open $a d$ hoc methodologies to bring a social adherence to the decisions. This statement is the overall motive of the SUIT project, a three-years research devoted to the sustainable development of urban historical areas through an active integration within towns, developed under the auspices of the European fifth research framework program,whose theme 'Energy, environment and sustainable development', Key action 4: The city of tomorrow and cultural heritage.

The present paper deliberately focuses on one specific objective of the SUIT project (for more information about the SUT project, please refer to: http://www.lema.ulg.ac.be/research/ suit/), namely to deliver an on-line glossary tool whose role would be to foster the construction of a common culture among urban experts, stakeholders and decision makers. First, we depict briefly urban conservation as increasingly confronted with a situation somewhat paradoxical. On one hand it is challenged by a loss of credibility of the different actors associated with the decision making, while on the other hand our scientific instruments and methods (sustainability indicators, visualization tools, conservation techniques etc.) and the available communication medias (mail, world wide web) have probably never been more efficient. Secondly, we review some technical solutions that were proposed to address this issue. Among these, the argumentation based support tools were probably the more promising ones. It should still be assumed that any collaborative decision making requires some common culture to make the dialogue possible and constructive. Such a common understanding of basic terms, references and methods is often missing in present-day urban disputes, especially when complex issues such as cultural heritage are at stake. We thus proposed the use of online glossaries as a way to settle these disputes. The conceptual aspects of such a system are then described, while the technical aspects of an existing on-line glossary prototype, developed during another European research project, follow.

\section{Problem statement}

It is probably during the recent food and health crises that the loss of credit suffered by experts and decision makers among the general public found its most acute expression. Still deep credibility crises are no longer unusual in important urban management projects, even though the geographical extent and the population involved obviously remain more limited. These are especially the case when heritage issues are at stake, and when the population is well aware of the irreversible character of the decisions that may be adopted in its name. It has also to be considered that cultural heritage usually involves different registers of values, may they be economical, cultural, social ecological or simply affective ones. It is these values that truly confer its symbolic character to the built heritage, whether it consists of isolated monuments or entire fragments of an urban pattern. Accordingly, public concern for these elements is not usually delimited to the area of a particular action and its consequences. It is apparent that such fundamental questions often overrule any kind of information in regard to 
having an impact on the course of the decision-making process. If questions which are important to the local public are underrepresented, and therefore a lack of information in regard to these 'soft' factors becomes evident in a dispute resolution procedure, it is often too late to start the mediation efforts.

Moreover, public-private partnerships have progressively become the rule for large urban development plans, since the combined pressures of decentralization and liberalization (Claydon \& Smith, 1997 ; Dupagne, 1996 ; Laterasse, 1996 ; Sagalyn, 1997). Such partnerships raise specific difficulties when applied to highly public and collective values, as is the case for much of our urban heritage. Private actors are not usually trusted to engage in such contentious situations alone, given the existing pressure of environmental groups. Still the public authorities no longer have the power to impose their own views to these economical actors. As a consequence, the built environment regulation often implies direct negotiations between the local authorities and the private developers so as to maximise so-called 'planning gains'. The legitimacy and credibility of the public intervention is further questioned by these negotiations, especially when they develop in camera as it is often the case.

The scientific and technical experts have also lost part of their credibility among the population (Institute for Prospective Technological Studies, 2001). Quite generally, the status of impartiality of science seems to be more and more contested. This is explained firstly through the acknowledged financial dependency of scientific actors towards political and economical spheres. We thereby assist to a progressive secularization of science, that is more and more considered as part and parcel of a larger social and political system. As such there is an increasing public awareness of the fact that experts are themselves subject to basic considerations of career, visibility or simple balance of power, as in all other social fields (Shackley et al., 1998). One notes, in counterpart, a sustained feeling of a necessary belief in trustable and independent knowledge. This claim was particularly acute during the recent European food crises, but it could also be observed in other decision-making fields (climate change, urban management, protection of the biodiversity). It has still to be considered that, by definition, the expertise often consists in going beyond proved scientific knowledge: if an expert is called to intervene in a controversy, it is most certainly due to the lack of definitive scientific answer to the given problem. Accordingly, the specific competencies of citizens to express a relevant opinion, even on such complex issues, should neither be disregarded. From this perspective, the deference, ignorance, scepticism or passive disinterest may all turn out to be deliberate forms of rejection of science, when it intents to impose its own interpretative frameworks (Michael, 1996).

Finally, the autonomy of lobbies and interest groups is nonetheless guaranteed. It is even observable that, if these new players have progressively gained the power to block certain developments, they usually miss a strong constructive capacity to formulate and support alternative urban conservation projects (Knoepfel, 1993).

To sum up, it seems urgent to develop new methods to improve the democratic control of political decision makers and urban experts by citizens. This implies first and foremost building a common culture, shared by experts, stakeholders and decision makers. Our purpose here is not to eliminate any form of controversy, but to create the conditions that would allow a positive resolution of divergent opinions. One of our basic assumptions is indeed that controversy and critical scepticism are to be considered as situations whose outcomes may be positive provided that they do not degenerate into an open conflict, or to irreconcilable positions. 


\section{Argumentation support tools}

There exists a number of technical resources to tackle the issues mentioned. Quite paradoxically, it may even be considered that the scientific and technical methods available to address the active conservation of urban heritage have probably never been more efficient. There is indeed a rapidly expanding knowledge of the way to assess the urban long-term sustainability (Expert Group on the Urban Environment, 1996). A comprehensive toolkit of the methods applicable to the assessment of sustainable urban development has been proposed in the framework of the European BEQUEST project (Deakin et al., 2001). It is based on a fourfold representation of sustainability (environment, equity, participation and futurity).

The available sets of sustainability indicators are taking into account an ever growing number of factors, among which the effects of urban development on material and immaterial heritage structures, through dose-response factors f.i. (CEC, 2000). These sets of indicators have been progressively included in the geographical information system (GIS) tools available on the market since the beginning of the nineties (May et al., 1996). Innovative representation methods were specifically developed to communicate the intentions of an urban conservation project (Dupagne and Teller, 1998) and the existing visualization tools are more and more efficient and affordable. Thanks to the outstanding development of the internet and communication infrastructures, groupware technologies can now be applied to the direct public involvement within the urban decision making, while it was mostly a privilege of technical actors until now (Laurini, 2001).

In the meantime, it has been broadly recognized that such methods and techniques would certainly benefit from a closer integration with argumentation support tools, in order to foster their genuine social appropriation. Tweed (1997) and Gottsegen (1998) proposed to adopt the model of the argument developed by Toulmin to formalize the structure underlying environmental disputes. Even though their approaches are mostly of a descriptive nature, they appear to be a very promising way to organise a reasoned debate between stakeholders, decision makers and experts. The use of information technologies to store and manage the arguments exchanged during the course of a controversy hence appears to be a very promising way to capture the rationale of the decision-making process.

The model of an argument proposed by Toulmin has the advantage of being highly formalized. As such, it fits very well with an integration into information technologies. Still its generic character is also its main weakness in that it does not leave much room for the argumentation context and the question of the public in particular. It has been acknowledged, since Aristotle and his Rhetoric, that the context plays a major role in the course of argumentation, in terms of process, values and genre. These ideas were central to Perelman's work. According to Perelman et al. (1958), argumentation is fundamentally different from logical reasoning due to the interrelation process that characterizes it: "the objective of an argumentation is not to deduce facts from premises, but to provoke the adhesion of a public to a given thesis presented to its approval'. This would lead Perelman and other researchers from this school of thought to focus their studies around how an argument is received.

Confronted with quite the same problem in the domain of multipurpose GIS, Harvey (1997) once suggested building upon the sociology of translation. This led him to propose a design methodology which first consists in identifying the concepts that are somehow shared between different technical cultures. These shared concepts, called boundary objects, may be geometries or attributes. More interestingly their definition can be common but also irreconcilably controversial between the involved cultures. The participatory approach he proposed 
consists precisely in the resolution of controversial definitions through the construction of morphisms, or 'portals', intended to assure the translation of an object from one worldview to another without forcing a common definition between the two worlds. This approach contrasts sharply with the attitude of some computer aided design (CAD) authors, like Zolin et al. (2000), who consider mutual trust as a basic condition for collaborative work. While it seems acceptable for reduced team of experts, sharing the same initial vision of the project, such a condition may be difficult to fulfil in some urban contexts. It is especially the case when development projects involve a conflict between significant stakes, interweaving different fields of values, like the personal and collective interest or material and symbolic aspects.

\section{The use of web glossaries}

We propose adopting an on-line glossary as a way to canalize and structure public controversies occurring during urban conservation projects. As can be understood from the previous discussion, the question here is not to eliminate conflict but rather to create the conditions which encourage a constructive resolution of the controversies at the early stage of the decision making process: exploration and structuring of the problem.

A first premise is that conflicts have to be approached as situations which may have positive effects, depending on how they are managed. A second premise is acknowledgement from the outset that these controversies are mixed-motive, ill-defined situations and therefore may be represented differently by the various stakeholders involved. Acknowledging the inherent subjectivity of any such representations implies that perceptions reported by all involved parties are to be treated as being equally valid at the very beginning of the decision making process. Yet the argumentation would benefit from a common basic vocabulary, in terms of methods, indicators or concepts. The above mentioned subjectivity of each actor is not incompatible with the existence of some concepts that may be shared by the different participants even though their definition sometimes remains controversial. Furthermore, when the explicit definition of a term raises a difference between the involved stakeholders, the controversy should be identified, acknowledged and eventually addressed before it turns into an intricate and destructive conflict. It has also been considered that any common culture would gain from being based on familiar situations, in order to demonstrate the pertinence and validity of the concepts through well known examples, controllable by the different actors of the decision making process. Systematic references to the local context, effectively experienced by the population, should drive support for a real social appropriation of the methods and techniques, rather than simply rely on the authority argument as was often the case by past.

Following this approach, a preliminary European project, called AUDIENCE, proposed to use an Internet site (http://www.lema.ulg.ac.be/tools/audience/) as an electronic platform, designed as a reference context for comparison and discussion. The proposed on-line glossary was understood as a dynamic, open and local system. Its kernel consists in a selective dictionary, which only deals with urban conservation. As such, it does not compare with the universalist encyclopediae, once conceived, in the eighteenth century, as the extensive sum of all available knowledge available at one point of history. Any actor, involved in a way or another in an urban conservation project, can find on this platform a dynamic cultural framework with different entry gates corresponding to different needs in understanding the domain and to get the best adapted information. An actual urban conservation project can be 


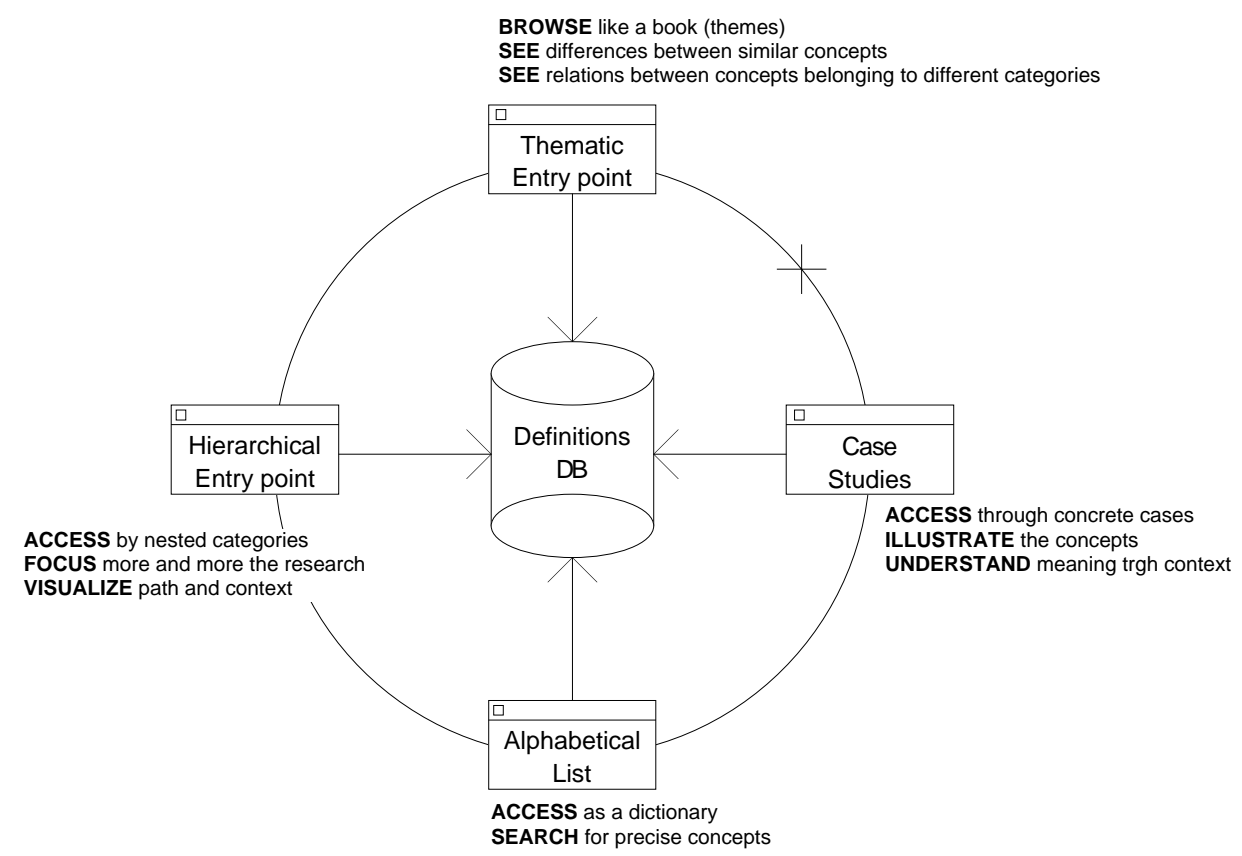

Figure 1 AUDIENCE conceptual organization

compared with past experiences specified by all their indicators available on the electronic platform, presented in an attractive and easy understandable way. The proposed on-line glossary is accessible through four different entry points (Figure 1).

The first entry point is a simple alphabetical list, organised as a traditional dictionary. It allows the rapid searching of a precise concept definition, phrased in quite technical terms. The use of this entry point requires the user to already have some familiarity with the concept and to search for more precise data (reference values of an indicator, detailed source etc.). Using this entry means a search for concept clarification. It is also the place where conflictual definitions can be stated and addressed. Each definition can lead to an exchange of arguments, since it has to be justified. This is especially the case for performance-based definitions that unavoidably carry with them a series of values, as in the case of sustainability indicators.

The second entry point is a thematic glossary, where concepts are classified along themes and subthemes. Each subtheme is a semantic unit which gathers a set of concepts that are somehow relevant to a given topic. This structure allows some form of analogical reasoning and easy access to technical terms by proximate, known concepts. The second entry point supports a more classical learning style, a book-like approach to the domain. For instance, the theme 'comfort' is composed of several subthemes: 'Stationary thermal comfort', 'Adaptive thermal comfort', 'Visual comfort' and 'Intimacy'. The subtheme 'Stationary thermal comfort' gathers concepts like 'iso-comfort graph', 'metabolism', 'activity level', 'skin temperature', 'direct radiation' etc. Themes and subthemes hence consist of higher-level semantic units, which allow to establish weak links between proximate concepts, may these be characterized by relations of 'similarity' or 'antonymy'. 


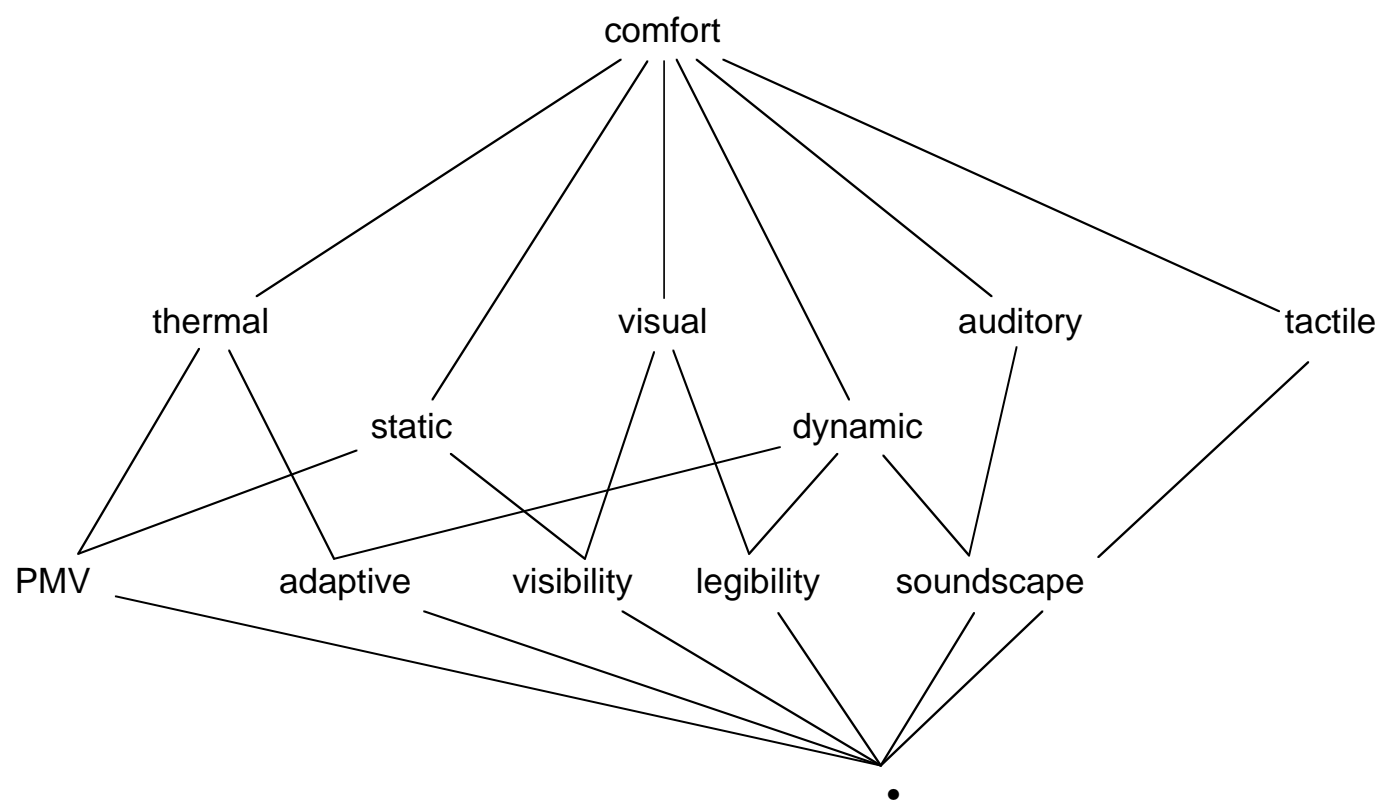

Figure 2 Hierarchy of categories deriving from the 'comfort' concept

The third entry point is a hierarchical structure which proposes a decomposition of the conceptual meaning along a specialization graph, ordered by the type-subtype relation. The resulting lattice of concepts provides some basic understanding of a concept's meaning by the deliberate definition of its direct filiations, in terms of parent and children nodes. This entry point allows a more divergent approach and a more standalone learning style.

Figure 2 provides an example of such a decomposition applied to the domain of 'Comfort'. It distinguishes between thermal, visual, auditory and tactile comfort. Each of these categories can then be understood in a static or a dynamic way. This supplementary distinction leads to further develop the graph, so as to distinguish between the approach of predicted mean vote (PMV) and the notion of adaptive comfort for instance.

It is well known that different persons may propose different decomposition of a same domain, according to the goal they pursue. Any systematic glossary should thus always be kept open to further refinement and completion (Sowa, 2000). It should for instance be possible to add a new dimension in the comfort graph so as to discriminate between 'indoor' and 'outdoor' concepts. The structure proposed in AUDIENCE was heavily focused on passive solar design, which constituted the major aim of the program. Yet more urban design-like structures may be proposed, on the basis on the same concepts, plus additional ones.

The fourth entry point consists of references which illustrate the concepts through their concrete application in a specific context. Such references are meant to give some idea of the value of indicators or the result of methods within external situations, whose relevancy to the urban project under consideration can then be discussed. A worked example based on the application of thermal comfort concepts and methods has for instance been integrated in the AUDIENCE Web site. It consists of the analysis of a public open space of the Expo'98 in Lisboa. 
Resorting to references also allows users to gain some familiarity about the way a specific concept may be used within an argumentation exercise, as illustrated by its critical application to a given issue. It is indeed well known that the meaning of methods and concepts often lies in their use rather than in their form (Harvey, 1997), especially when controversial notions are at stake. The references discussed in the glossary have thus to be clearly distinguished from 'cases', once defined as patterns to be somehow followed, possibly after a process of abstraction. By contrast with such cases, the references in the glossary may be positive or negative according to the way they are themselves mobilized in the arguments. That is to say that their quality is not intrinsic. Rather the distinctive property of such references is that they have to benefit from a status of proved facts: their relative value along given dimensions is to be recognized as such by the different tenants of the discussion. This justifies the use of local examples, well known by the local public. Alongside the example of Lisboa'98, the analysis of a local example considering a city entrance of Liège (Belgium) has for instance been integrated in the database, so as to give an idea of the concrete meaning of concepts like visual saturation, visual noise and lack of legibility.

The four entry points are connected through a set of hyperlinks, which allows to rapidly pass from one view to another. Each definition contains links to themes, hierarchies and references it is related with. It is possible to go directly to the definition of any concept mobilized by a reference. Themes and hierarchical structures are basically sets of links to the definitions.

\section{Technical implementation}

The on-line glossary has been developed by using a dynamic connection with a simple and easy to manipulate Access database. The system is entirely based on the active server pages (ASP) technology. Some specific software modules, required for the interaction between the Microsoft Internet Server and the Access database, were developed in Delphi. In this way, all the on-line glossary pages and hypertext links can be dynamically generated on the server side according to the database queries formulated when browsing through the ASP pages.

The database conceptual model is roughly summarized in Figure 3. The entire structure of the on-line glossary is stored in the Access database as tables of themes, subthemes, references and nodes, as well as relations between them. A node can consist of an indicator, a method or a concept. Each node can be related to one or many nodes and associated with one or many textual definitions, which are themselves associated with one author and one or many illustrations. The references are described in the database by a title, the URL (uniform resource locator) of a static HTML (hypertext markup language) page describing the case and links to concepts. One concept may appear in many references and references may obviously refer to many concepts. The many-to-many relation table, relating references and concepts is automatically updated, by a specific application program interface (API) parsing the document each time a new reference is added into the system, or when a reference is modified.

Some 324 nodes have been experimentally introduced in the system. Among these, 239 nodes received a precise definition, provided either by ourselves or the existing literature. These definitions are classified according to 10 themes and 46 subthemes, ranging from the concept of the 'urban box' to the measurement of daylight accessibility (see Figure 4). A hierarchical tree has been developed and five cases have been integrated in the system. These five cases are based on previous research works :

- morphological analysis of a central urban space (Place Saint-Lambert, Belgium); 


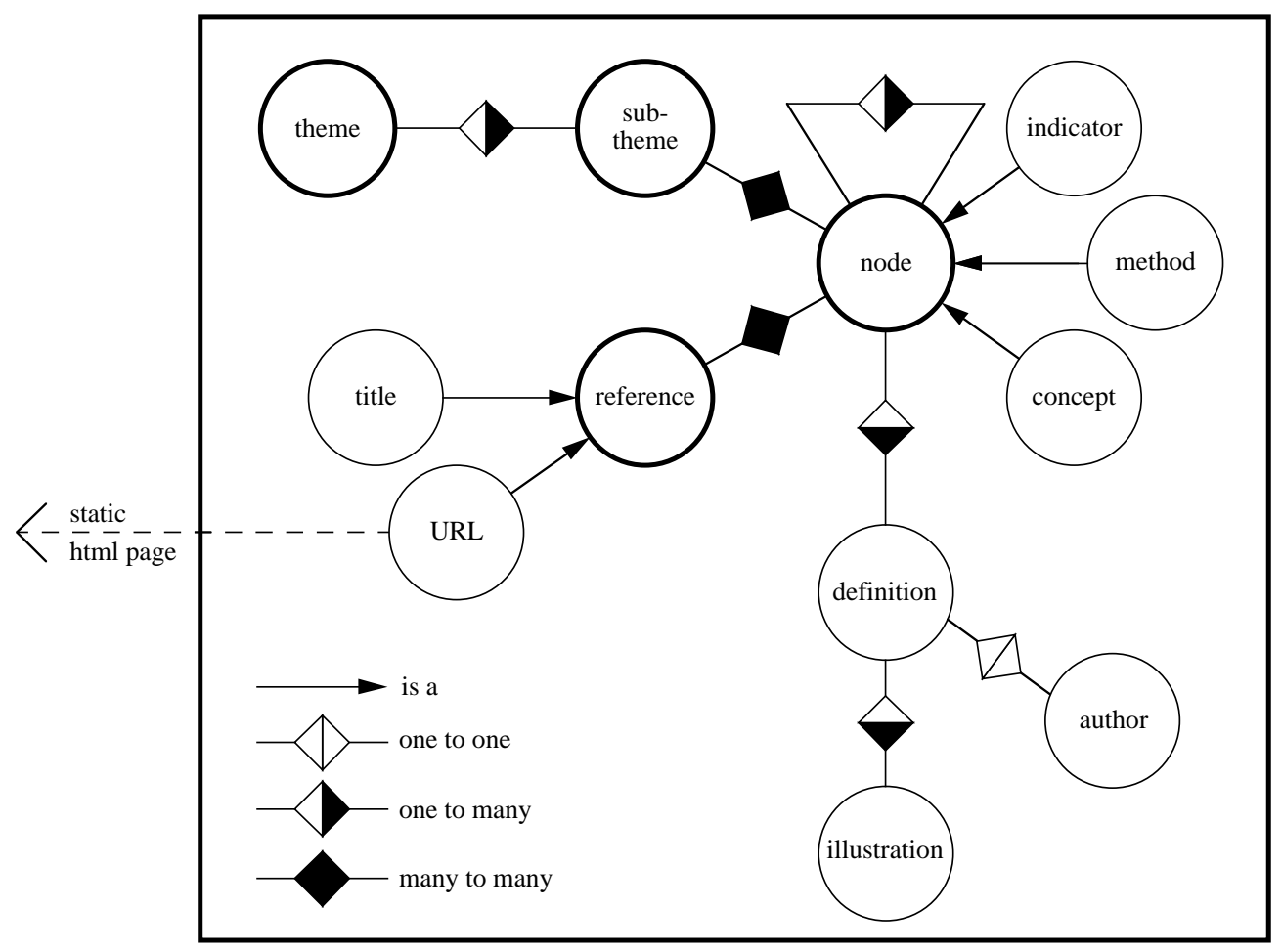

Figure 3 Entity-Relationship model of the database

- thermal comfort within open spaces of the Lisboa'98 International Fair (Lisboa, Portugal);

- landscape analysis of a small rural village (Marchin, Belgium);

- visual saturation along a city entrance (Liège, Belgium); and

- internal accessibility graph of a very dense urban block (Liège, Belgium).

These cases were basically selected for their diversity so as to enlarge the scope of the glossary. This initial selection should obviously be completed to consolidate the kernel of the system.

As the AUDIENCE project concerned on-line teaching for architects, the on-line glossary prototype has been reviewed by five Belgian end-users trained in architecture. They were all involved in the professional practice, even though with different levels of experience. Their comments allowed a progressive fine-tuning of the system. This validation mechanism should now be enhanced and enlarged to other groups of end-users within the SUIT project. It would be based on an application to an actual case of urban controversy.

The on-line glossary prototype is accessible, in French, at the following URL address: http://www.lema.ulg.ac.be/tools/audience.

\section{Discussion}

If an argumentation is to be developed so as to progressively gain a genuine support from its 


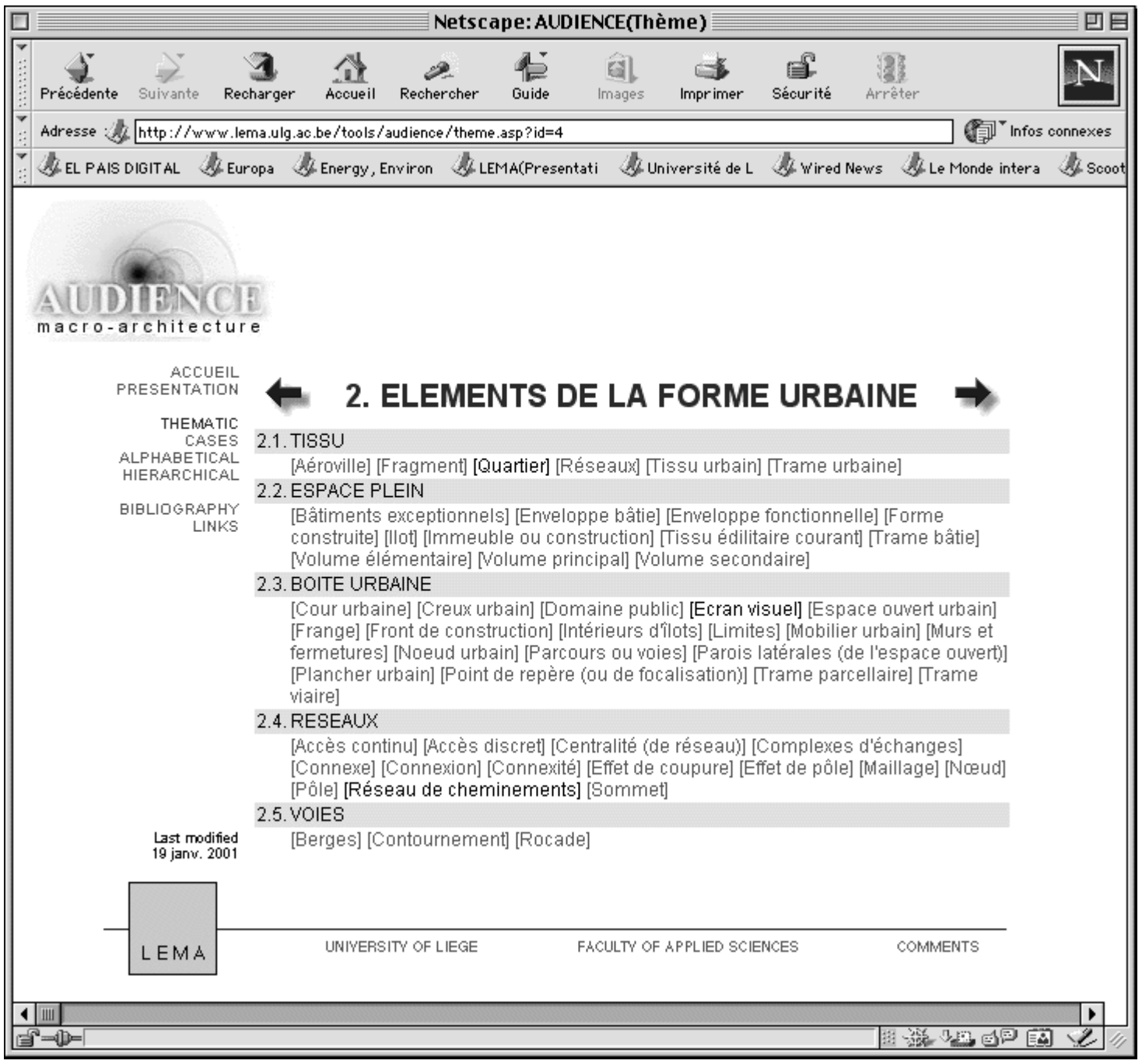

Figure 4 Snapshot of the second entry point (thematic glossary)

participants, it is necessary to build up some form of common culture, or at least a shared meaning of concepts, indicators and methods, accepted by the decision makers, the experts, the stakeholders and the population. The communication facilities available at present probably constitute an outstanding opportunity in this regard. Still such an utilization of the world wide web, as a common culture building tool, has not yet been the object of many researches, especially in the field of urban conservation. It should indeed be considered that this field of expertise and training has traditionally been striking for a greater acknowledgment of its scientific specificity and legitimacy. An increased consideration of the population claims into the debates surrounding the conservation of heritage is not always well accepted by the experts (Dupagne et al., 2001). This is for sure an element to be taken into consideration when a more constructivist approach of the public controversies is advocated for. Hence our 
view basically implies considering the perceptions and representations of the public and the stakeholders on the same ground as the one of experts and trained specialists at the beginning of any argumentation practice.

Our contribution to this issue is strongly exploratory, and the web site we presented remains a prototype, solely developed for experimental reasons. The on-line glossary still functions in 'closed' mode. Just as now, it is not yet possible for an external user to feed directly the system with new definitions, new themes or new references. Technically, given the flexibility of the ASP, it would be no great challenge to allow users to fill in html forms and dynamically integrate the provided information within the database. The problems raised by this issue are rather practical. It would indeed require to first settle how to manage the conflicts between different definitions or within a definition, how to insert a new node in the system, how to authorize the generalization of a concept and who will have the power to control entries to the glossary. Also it should be decided how and when a consensus has been reached around a given node, or more difficult, around a specific branch of a tree.

It can hence be considered that the vertical axis of Figure 1 is mostly characterized by a convergent approach in the project. These entry points assume some agreement around the existence of certain concepts and their thematic organisation. By contrast, the horizontal axis is characterized by a more divergent and dynamic nature. This appears very important to allow real innovation and local instantiation of the system. Yet it has to be recognized that any conservation project requires some stability to develop: stability of the terms of reference, of the applicable rules and expected results. This implies that the system could not be opened before some better definition had been found on how to stabilize some of its components and how to transfer part of a hierarchical structure to a thematic structure.

Another limitation of the present system lies in the fact that the glossary is solely based in French. Its further extension to other languages would raise challenging questions as soon as one would attempt to develop some form of multilingual index. Actually the notion of "urban design' does not have any straightforward translation in French for instance. It is proximate to the notions of projet urbain and composition urbaine without being synonymous of any of these terms. The projet urbain (urban project) is indeed usually considered as a socio-political construct. Its definition rather insists on the public nature of the urban realm while the composition urbaine (urban composition) tends to be more heavily focused on the formal and geometrical properties of the space, in terms of vistas and alignments.

The EuroWordNet project consisted of a multilingual database combining eight different European languages (Vossen et al., 1999). An inter-lingual index (ILI) was therefore developed, based on prior experience of WordNet 1.5 which was further refined to accommodate supplementary sense-differentiations. Even though quite theoretical, the experience gained during this project probably constitutes a valuable starting point for the building of a crossEuropean urban glossary.

Finally, it has to be stressed that if our objective is to facilitate the argumentation and, thereby, canalize the controversy, we think that it is also able to enlarge the circle of the people involved in the decision-making process, may it be to criticize it or to defend it. We hereby refer to the idea, developed in our problem statement, that claims that passiveness and resignation may all be positive forms of contestation of a discourse that would be considered as excluding. The idea to construct a common culture as proposed in this paper, and more generally in the SUIT project, in parallel or in advance of the argumentation process, is intended to reduce this type of reaction, like the false consensus, the passive resignation or the deference. 


\section{Acknowledgements}

This paper is based on experience gained during the AUDIENCE project, co-ordinated by the CERMA (School of Architecture, Nantes). The AUDIENCE project was supported by the EU Program LEONARDO DA VINCI. The AUDIENCE contract number was F/96/1088/PI/ II.1.1.c/FPC.

A follow-up of this exploratory research is presently developed in the framework of the SUIT project - sustainable development of urban historical areas through an active integration within towns. The SUIT project is supported by the EU Program 'Environment and Sustainable Development', Key Action 4 : The City of Tomorrow and Cultural Heritage, Theme 4.2.3 : Foster Integration of Cultural Heritage in the Urban Setting. The SUIT contract number is EVK4-CT-2000-00017.

\section{References}

CEC. 2000: A review of the European commission research on environmental protection and conservation of the European cultural heritage. European Commission.

Claydon, J. and Smith, B. 1997: Negotiating planning gains through the British development control system. Urban Studies 34, 2003-22.

Deakin, M., Curwell, S. and Lombardi, P. 2001: Sustainability assessment of urban development plans. Proceedings of BEQUEST - Lisboa workshop, 26 April .

Dupagne, A. 1996: Urban processes and decision making. In Schiavoni, U., editor, Proceedings of International workshop on information systems and processes for urban civil engineering applications, Rome, 21-22 November, 3-13.

Dupagne, A. and Teller, J. 1998: Spherical projections as a communication instrument for morphological decision-making in urban design. In Laurini, R., editor, Proceedings of international workshop on groupware for urban planning, Lyon, 4-6 February.

Dupagne, A., Rogister Y., Ruelle, C. and Zwetkoff, C. 2001: SUIT Task 1.1 ReportExploratory defnition of the end-users' requirements. Internal Report. LEMA, University of Liège.

Expert Group on the Urban Environment. 1996: European Sustainable Cities, European Commission, DG XI, environment, nuclear safety and civil protection, Brussels, March 1996.

Gottsegen, J. 1998: Assessing the interests and perceptions of stakeholders in environmental debates through argumentation analysis. In Laurini, R., editor, Proceedings of international workshop on groupware for urban planning, Lyon, 4-6 February.

Harvey, F. 1997: Improving multi-purpose GIS design: participative design. In Hirtle, S. and Frank, A.U., editors, Proceedings of COSIT '97 - Spatial Information Theory: A Theoretical Basis for GIS, 313-28.

Institute for Prospective Technological Studies. 2001: Special Issue: Science and Governance in a Knowledge Society. IPTS Report, 55.

Knoepfel P. 1993: Approaches to an effective framework for environmental management. Cahiers de l'IDHEAP, 108a.

Laterasse, J. 1996: La gestion urbaine: de nouveaux défis pour les aménageurs? In L'aménageur urbain face à la crise de la ville, éditions de l'Aube, 159-71.

Laurini, R. 2001: Information systems for urban planning. London: Taylor and Francis.

May, A.D., Mitchell, G. and Kupiszewska, D. 1996: The quantifiable city: the development 
of a modelling framework for urban sustainability research. In Schiavoni, U., editor, Proceedings of COST UCE Action C4 conference on information systems and processes for urban civil engineering applications, Office for Official Publications of the European Communities, EUR 18325 EN, 124-42.

Michael, M. 1996: Ignoring science: discourses of ignorance in the public understanding of science. In Irwin, A., Wynne, B., editors, Misunderstanding science? The public reconstruction of science and technology. Cambridge: Cambridge University Press.

Perelman, C. and Olbrechts-Tyteca, L. 1958: Traité de l'argumentation, la nouvelle rhétorique. Paris: Presses Universitaires de France.

Shackley, S., Darier, E. and Wynne, B. 1998: Towards a 'folk integrated assessment' of climate change. In ULYSSES Working PaperContribution to participatory integrated assessment, ULYSSES WP-98-1, Internal Report, Darmstadt, Germany: Darmstadt University of Technology, 1-24.

Sagalyn, L.B. 1997: Negotiating for public benefits: the bargaining calculus of public-private development. Urban Studies 34, 1955-70.
Sowa, J.F. 2000: Knowledge representation: logical, philosophical, and computational foundations. Pacific Grove: Brooks Cole Publishing.

Teller, J. 2001: La régulation morphologique dans le cadre du projet urbain. $\mathrm{PhD}$ thesis presented in the University of Liege.

Tweed, C. 1997: An information system to support environmental decision making and debate. In Brandon, P.S., Lombardi, P.L. and Bentivegna, V., editors, Evaluation of the built environment for sustainability. London: E \& FN Spon, 67-81.

Vossen, P., Peters, W. and Gonzalo, J. 1999: Towards a universal index of meaning. In Proceedings of the ACL-99 Siglex workshop. University of Maryland.

Zolin, R., Fruchter, R. and Levitt, R. 2000: Building, maintaining and repairing trust in global AEC teams. In Fruchter, R., PeñaMora, F. and Roddis, W.M.K., editors, Proceedings of the eighth ICCCBE Conference - Computing in Civil and Building Engineering, Stanford, 14-16 August, 874-81. 